\title{
Improving the oral bioavailability of beneficial polyphenols through designed synergies
}

\author{
Arjan Scheepens $\cdot$ Kee Tan $\cdot$ James W. Paxton
}

Received: 22 July 2009/Accepted: 31 August 2009/Published online: 20 October 2009

(C) Springer-Verlag 2009

\begin{abstract}
A substantial and growing consumer demand exists for plant-based functional foods that improve general health and wellbeing. Amongst consumed phytochemicals, the polyphenolic compounds tend to be the most bioactive. Many commonly consumed polyphenols have been shown to have specific and potent health-promoting activities when assessed by high-throughput in vitro assays and when administered to experimental animals by injection. However, very few have been shown to have any beneficial effects in animals or man when orally consumed, because of the poor bioavailability exhibited by most polyphenols following the ingestion. Consumed polyphenols, like most pharmaceuticals, are regarded as xenobiotics by the body and must overcome many barriers, including extensive enzymatic and chemical modification during digestion and absorption, to reach their site(s) of action. This is especially true for polyphenols targeting the brain, which is protected by the tightly regulated blood-brain barrier. Interestingly, many polyphenols are also known to specifically modify
\end{abstract}

This article belongs to a special issue on the 4th international Niigata symposium on diet and health, 29-30 November 2008.

A. Scheepens $\cdot$ K. Tan

Functional Foods and Health, The New Zealand Institute for Plant and Food Research Limited, Mount Albert, Private Bag 92-169, Auckland Mail Centre, Auckland 1142, New Zealand

J. W. Paxton

Department of Pharmacology and Clinical Pharmacology, Faculty of Medical and Health Sciences, The University of Auckland, Auckland, New Zealand

A. Scheepens $(\bowtie)$

Plant and Food Research, 120 Mount Albert Road, Mount

Albert, Auckland 1025, New Zealand

e-mail: arjan.scheepens@plantandfood.co.nz some of the metabolic and transport processes that govern bioavailability. Therefore, the opportunity exists to increase the bioactivity of beneficial polyphenols by designing specific synergistic interactions with polyphenols that improve their oral bioavailability. This hypothesis and review paper will discuss some of the endogenous systems that limit the bioavailability of ingested polyphenols to the body and the brain, and the means by which bioavailability may be improved by specifically designing synergies between orally consumed polyphenols.

Keywords Bioavailability · Phases 1 and 2 metabolism . ABC transporters $\cdot$ Polyphenol $\cdot$ Synergy $\cdot$ Functional food

\section{Introduction}

Evidence from many studies has linked the intake of fruits and vegetables, with a diverse array of health benefits. This, alongside traditional plant-based medicines, has created a significant market for plant-derived functional foods which impart health benefits. This demand is further driven by an increasing consumer awareness of health, the benefits of a healthy diet, and the reluctance to depend on pharmacological intervention. Classically, the science behind the creation of functional foods has involved taking extracts with putative health benefits, either from epidemiological data or traditional-medicine-based evidence, and subjecting these to high-throughput assays on specific molecular targets in vitro. Activity-guided fractionation is then used to identify bioactive components that are purified, or enriched to form a functional food product. Many functional foods are now available which were designed in this way, but few have been subjected to rigorous clinical trials and many are sold on the basis of in vitro evidence only. For example, within the arena 
of functional foods that affect mood, true clinical efficacy has only been shown for a handful of plant extracts, including kava kava for anxiety [64] and Salvia officinalis for improving mood and cognitive performance [34, 77].

Plant-based food are comprised of widely varied compounds including, fibres, vitamins, minerals, ions, carbohydrates and polyphenols, all of which have some functionality within the body. Increasingly however, it is the polyphenolic compounds that are thought to be the most bioactive agents within plant-based foods. These are categorised into several classes, namely: hydroxybenzoic acids, hydroxycinnamic acids, anthocyanins, proanthocyanidins, flavonols, flavones, flavanols, flavanones, isoflavones, stilbenes and lignans [43]. By far, the major issue in the development of bioactive polyphenols and food ingredients is their limited bioavailability, and many excellent reviews have been written on this [42, 43, 75]. Using high-throughput in vitro assays followed by activity-guided fractionation however, does not take into account in vivo bioavailability and can lead to both falsepositive- and false-negative interpretations. For example, if the in vitro active compound within an extract is quickly metabolised or has limited bioavailability to the target organ, false-positive hits are found, and conversely, if the active compounds are actually metabolites of the compound(s) within a crude extract, then their bioactivity is not observed in vitro. It is clearly unethical and unfeasible to use in vivo models for high-throughput screening programmes and thus an appreciation of the mechanisms involved in the bioavailability of polyphenols is important. In general, the body treats consumed polyphenols as xenobiotics, or foreign substances, similar to most pharmaceuticals and these are subjected to the same protective xenobiotic-metabolisingand efflux mechanisms. This often results in major changes in biological activity and usually greatly increased rates of excretion from the body. Interestingly, many polyphenols appear able to modulate the mechanisms that govern xenobiotic bioavailability and putative synergies may be designed to increase the limited bioavailability and thus bioactivity of some xenobiotics.

In this review and hypothesis paper, we will first discuss the basic processes which govern the absorption, metabolism and distribution of polyphenols. We will then discuss some specific examples of synergies between polyphenols, which increase bioavailability of the bioactive and suggest future synergies which may be designed in the creation of more efficacious functional foods.

\section{Absorption, metabolism and distribution of polyphenols}

Polyphenols are mainly small organic molecules (molecular weight typically in the 200-800 Da range) with one or more phenolic ring structure. To produce their beneficial effects, other than on the gastrointestinal (GI) tract itself, these xenobiotic molecules must be absorbed into the body after oral ingestion and be carried by the blood stream from the absorption site to target tissues and organs. Biological activity has been demonstrated for many of these polyphenols in numerous in vitro systems [75], but it is apparent that the effective concentrations $(>\mu \mathrm{M}$ levels) in vitro are at least an order of magnitude higher than those $(<1 \mu \mathrm{M})$ normally achieved in human plasma [43]. To achieve sufficient concentrations at their site(s) of action, consumed polyphenols must overcome a number of barriers. First, they must dissolve in the fluids of the GI tract and survive the low-pH environment encountered in the stomach. They may also be subjected to degradation and metabolism by intestinal enzymes, such as the glycosidases, esterases, oxidases and hydrolases originating both from the host and the myriad of microbiota that inhabit the GI tract [71]. One group of gut enzymes, the monoamine oxidase (MAO) enzymes, deaminate dietary monoamines and are a potential target for the design of synergies as discussed in "Modulation of gut MAO enzymes".

Most of the GI microbiota resides in the large intestine and the rate and extent of metabolism by bacteria will be influenced by the amount of the polyphenol that reaches the distal gut. Metabolism of polyphenols by GI enzymes may be responsible for the destruction of their bioactivity, but this is not always the case. For example, many plant flavonoids exist in the $O$-glycoside form within the plant and undergo hydrolysis to form their respective aglycons $[10,82]$. The latter are more lipophilic and more permeable across the cell membrane than the parent glycoside, and thus are more efficiently absorbed across the GI wall. However, favourable absorption across the gut wall (usually by passive diffusion down a concentration gradient or by an appropriate uptake transporter) does not always equate with improved bioavailability. Once in the enterocyte, the polyphenol or other xenobiotic may be subjected to various efflux pumps including the ATP-binding cassette (ABC) transporters, P-glycoprotein (Pgp/ABCB1/MDR1), multidrug resistance-associated protein 2 (MRP2/ABCC2) and breast cancer resistance protein (BCRP/ABCG2), which actively transport the polyphenol (or its metabolites) back into the GI lumen [30].

The ABC transporters are mostly found in biological membrane barriers including the epithelial layers of the GI tract, kidney, liver, placenta, blood-brain barrier (BBB), testes and choroid plexus. These transporters are well known for their ability to limit the bioavailability of pharmaceutical agents and evidence suggests that several clinically relevant food-drug interactions occur because of the ability of some food components to either upregulate or inhibit the efflux pumps, causing reduced drug bioavailability and efficacy, or higher absorption and possible toxic 
side effects, respectively (reviewed in detail in [56]). These are discussed in greater detail in "Polyphenols as transporter substrates" below.

Possibly of greater importance as a defensive barrier against these invading foreign molecules is the battery of both phases 1 and 2 enzymes present in the enterocytes. The phase 1 reactions include oxidation, reduction and hydrolysis, which primarily serve to increase the hydrophilicity of the molecule, and expose or add a functional group (such as a hydroxyl group) to facilitate phase 2 conjugation reactions. Oxidation is the most predominant reaction involved in the phase 1 metabolism of xenobiotics, and is principally carried out by a family of closely related isozymes known as the cytochrome P450-dependent mixed-function oxidases (CYPs). In humans, CYP1A, CYP2C, CYP2D and CYP3A are responsible for metabolising the bulk of xenobiotics that enter the body via the oral route [38]. CYP3A4/5 with its broad substrate specificity is particularly important in xenobiotic metabolism, making up 70 and $30 \%$ of total CYPs in the intestines and liver, respectively [94].

The parent polyphenols (or their phase 1 metabolites) that contain suitable functional groups (e.g., a hydroxyl group) often undergo conjugation reactions with endogenous compounds to yield more polar and water-soluble compounds. The latter are usually ideal substrates for active transport out of the cell, and eventually excretion from the body. The principal conjugation reaction is the formation of $\beta$-glucuronides catalysed by a family of enzymes known as the uridine diphosphoglucuronosyl transferases (UGTs), but conjugation with a sulpho moiety $\left(\mathrm{SO}_{3}{ }^{-}\right)$or glutathione also occurs, catalysed by various sulphotransferases (SULTs) and glutathione- $S$-transferases, respectively. Less-polar conjugates may also be formed by methylation, catalysed by catechol- $O$-methyl transferase. These phase 2 conjugation reactions are particularly important for polyphenols such as epi-gallocatechin-3gallate (EGCG), which is the most abundant catechin in green tea. EGCG has numerous hydroxyl groups and undergoes extensive phase 2 metabolism, including glucuronidation, sulphation and methylation [37, 88]. Several recent studies using liquid chromatography-tandem mass spectrometry (LC-MS/MS) have demonstrated that after ingestion of flavonoids, phase 2 conjugates of the aglycon such as glucuronides, sulphates and methylated metabolites predominate in the blood circulation, rather than the original plant glycoside or aglycon $[32,93]$. The extent to which these metabolites contribute to the overall beneficial effects of polyphenols in the body is largely unknown, and needs further investigation. In studies with green tea polyphenols, the metabolites mostly had reduced biological activity, but in some systems the metabolites had the equivalent or greater activity than the parent polyphenol
[37]. There is also evidence that the position of the glucuronide conjugate on the flavonoid can influence its biological activity [12, 33]. Certainly, these conjugated metabolic products are ideal substrates for various active transmembrane transport processes, in particular the excretory processes of the liver and kidney.

Although the phase 1 and 2 metabolic enzymes are found in the endothelial cells of the gut wall, by far the greatest concentrations are found in the liver, where they form a major barrier to the further distribution of the active moiety to other organs, such as of the heart, kidney, lungs and brain. The liver's location and the portal venous blood supply from the intestines make it well suited for the protection of the body from possible toxic xenobiotics contained in our diet. During this first passage through the liver, many polyphenols will undergo substantial extraction and metabolism (known as first-pass metabolism). The resulting metabolic products are then exported back out of the liver into the blood stream and carried to the kidney where they are excreted in the urine. Alternatively, metabolites such as glucuronide conjugates may be exported in the bile and released into the gut lumen. Thereafter, the metabolite conjugate may be excreted in the faeces, or alternatively it may be further metabolised by microbial enzymes, such as $\beta$-glucuronidase, which has the ability to cleave off the glucuronide and reform the lesspolar aglycon, which may then be reabsorbed. This cycle is known as enterohepatic recirculation and may result in a longer exposure of the body to the polyphenol. Evidence for such enterohepatic recirculation has been obtained for the flavonoid baicalein 7-O-glucuronide with a rat model [87], but whether a similar process occurs in humans for some flavonoids is not known.

Presumably, if the polyphenol overcomes the defence mechanisms of the gut and the liver, it will enter the systemic circulation and be distributed by the bloodstream to the other major organs of the body and possible site(s) of action. In pharmacology, the term bioavailability is used to indicate the relative amount of the ingested parent xenobiotic that reaches the main cardiovascular circulation (Fig. 1). Bioavailability is usually measured by taking peripheral blood samples over a period of time after ingestion and analysing for xenobiotic concentration. It is assumed that this blood concentration is an acceptable index for the concentration or exposure at the site of action. Polyphenols can pass with the ease through the pores of the capillaries of organs such as the heart and lungs, but not the brain. The brain capillaries are surrounded with a protective cellular sheath of glial cells (the so-called BBB) resulting in permeability characteristics more closely resembling those of tightly bound tissue cell walls. To gain access to the brain, a polyphenol must be highly lipidsoluble, or subject to uptake transport processes. In 
addition, the $\mathrm{BBB}$ contains a number of $\mathrm{ABC}$ transporter efflux pumps, which are involved in reducing the penetration of xenobiotics into the brain and protecting it [90]. It is apparent that there is a complex interplay between the physicochemical properties of polyphenols and the processes of metabolism and active transport that control their extent of exposure to their bioactive site(s) in the body. From experience with pharmaceuticals, it would appear feasible to manipulate these processes to obtain an improvement in bioavailability and greater exposure at the site(s) of action, to produce greater health benefits from appropriate polyphenols.

\section{Designing synergies to improve the bioavailability of polyphenols}

In this section, we aim to illustrate different strategies that may be useful in improving the bioavailability and hence bioactivity of polyphenols, and to give examples of such synergies where these are known. Given the tightness and relative impermeability of the BBB to polyphenols, we have mainly used examples of brain-active compounds to illustrate the possible effects of increasing the bioavailability of these plant-derived compounds.

\section{Modulation of gut bacterial metabolism}

Metabolism of dietary polyphenols by gut bacteria may constitute a significant barrier to their bioavailability. Chlorogenic acid, for example, is a very commonly consumed polyphenol present at relatively high levels in coffee and fruits, with the average person consuming up to $380 \mathrm{mg}$ per day [66]. Bouayed et al. showed that $20 \mathrm{mg} / \mathrm{kg}$

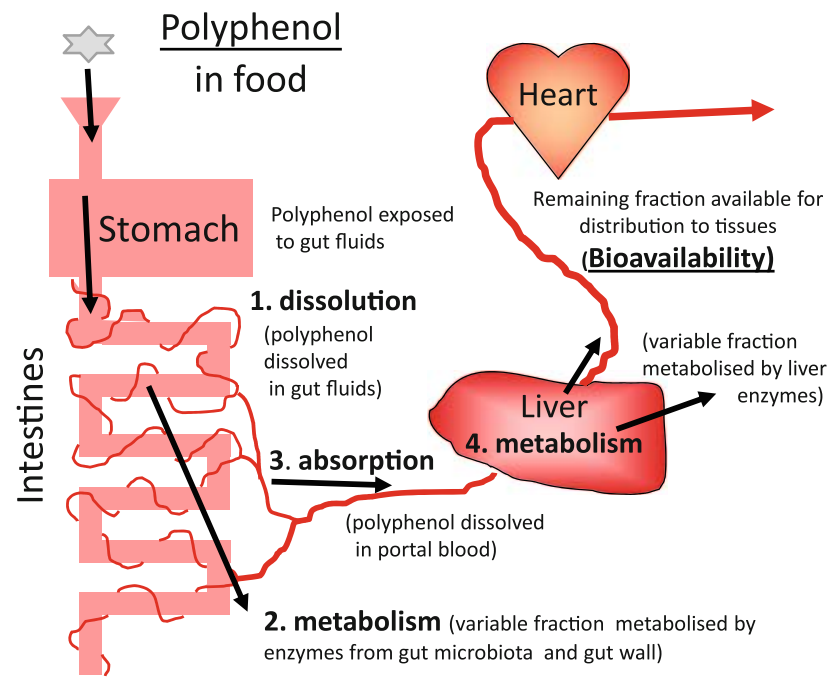

Fig. 1 Schematic representation of the major processes governing polyphenol bioavailability in mammals chlorogenic acid injected intraperitoneally into mice was as potent an anxiolytic as $1 \mathrm{mg} / \mathrm{kg}$ diazepam $\left(\right.$ Valium $^{\mathrm{TM}}$ ). Furthermore, its activity could be blocked by co-injection of the benzodiazepine receptor antagonist flumazenil, indicating that chlorogenic acid acts via the benzodiazepine binding site of the GABA-A receptor in vivo [5]. Thus, one might wonder why apples, coffee, prunes and cherries, all of which contain significant amounts of chlorogenic acid, are not known as anxiolytic agents. This is most likely due to the limited bioavailability of chlorogenic acid through the GI tract. Chlorogenic acid is a phenolic acid ester of quinic and caffeic acids, but only very limited amounts are found in plasma after the consumption of polyphenol-rich meals [50]. Most consumed chlorogenic acid is hydrolysed into the non-anxiolytic caffeic and quinic acids by the activity of bacterial cinnamoyl esterases in the colon, as well as esterase activity of host origin [1]. The resulting phenolic acids are relatively well absorbed via both active and passive transport processes, but are devoid of any benzodiazepine agonist activity.

Because gut esterase enzymes of both bacterial and host origin appear to be the primary mechanisms in the deactivation of chlorogenic acid, their inhibition may allow chlorogenic acid to remain in the GI tract for longer and thus be absorbed to a greater degree by passive diffusion. Esterase inhibitors, and specifically acetylcholinesterase inhibitors, are the primary target in the search for functional phytochemicals which may alleviate some of the symptoms of Alzheimer's disease and the cognitive decline associated with ageing. Hence, many potent and relatively non-toxic esterase inhibitors have been identified within the plant kingdom [55]. Given the target organ for this activity is the gut, the bioavailability of the orally consumed esterase inhibitors should be relatively unhindered.

A good example of a designed synergy to prevent bacterial degradation of a beneficial compound in pharmacotherapy is that of the broad spectrum antibiotic Augmentin $^{\mathrm{TM}}$. This antibiotic works on the principle of a sacrificial or "suicide compound". The primary antibiotic is amoxicillin, which has a $\beta$-lactam structure and is a substrate for the gut bacterial $\beta$-lactamase enzyme, which inactivates it. The other ingredient within Augmentin ${ }^{\mathrm{TM}}$ is clavulanic acid which also has a $\beta$-lactam structure and is also a substrate for the $\beta$-lactamase. The key to this synergy is that clavulanic acid is the preferred substrate for the bacterial $\beta$-lactamase binding irreversibly, and thereby inactivating the $\beta$-lactamase. This allows amoxicillin to escape degradation and be absorbed to impart its antibiotic properties. This mechanism also allows antibiotic activity against $\beta$-lactamase expressing, and thus amoxicillinresistant, bacteria (see [73] for review).

We were unable to find a polyphenol example of this type of designed synergy using sacrificial compounds. It is 
clear, however, that this type of synergy works well in pharmacotherapy and it should be possible to apply such a strategy to improve the bioavailability of a beneficial polyphenol which is degraded by gut enzymes of bacterial or host origin.

\section{Modulation of gut MAO enzymes}

A good example of a designed synergy between polyphenols leading to increased bioavailability and bioactivity through the inhibition of GI metabolic processes is provided by the traditional medicine Ayahuasca. This preparation has hallucinogenic properties and is used in shamanistic and religious rituals by South American Amazon peoples. It comprises two plants, each containing specific bioactives. First, the leaves of Psychotria viridis are used. These contain the substituted tryptamines $\mathrm{N}, \mathrm{N}$ dimethyltryptamine and 5-methoxy-dimethyltryptamine, both of which are serotonergic (5HT-2A) agonists with potent hallucinogenic properties [23]. Consumed alone, these monoamine compounds are susceptible to deamination by gut-derived MAO-A enzyme and their bioactivity is lost. However, when this extract is combined with the bark of Banisteriopsis caapi, a plant containing the powerful and reversible MAO inhibitors harmine and harmaline, the substituted tryptamines are protected from deamination by MAO-A and a powerful hallucinogenic experience, lasting up to 3 days, results [48].

This paradigm could be translated for the creation of synergistic functional foods. For example, chocolate is known to contain relatively small amounts of the monoamine phenylethylamine, which is also susceptible to deamination and thus deactivation by visceral MAO enzymes. Many commonly consumed plants have been shown to possess MAO inhibitory activity, including red wine, grapes, avocados and blackcurrants [4, 24].

Unfortunately, MAO inhibitors have deleterious side effects and are becoming less popular in psychiatry because of what is known as the "cheese reaction". Dietary tyramine, derived from fermented tyrosine and present in foods including aged cheeses, processed meat products and some fruits, can induce a hypertensive crisis and related migraines in sensitive people. The consumption of pharmaceutical irreversible MAO inhibitors can precipitate this effect by preventing the deamination of dietary tyramine within the GI tract. This side effect can be prevented by the use of reversible MAO-A inhibitors, able to be displaced from MAO-A by tyramine, or the use of selective MAO-B inhibitors which leave the visceral MAO-A free to deaminate dietary tyramine (see [91] for review). Patients who do not have the freedom to choose such MAO inhibitors are recommended to follow an MAO-I-diet, and avoid the consumption of the tyramine-rich foods [20]. Examples of both reversible MAO-A inhibitors and selective MAO-B inhibitors are known within the plant kingdom [24]. For example, quercetin is a relatively ubiquitous polyphenol found in many edible plants and has been shown to be a reasonably potent and selective MAO-A inhibitor with an $\mathrm{IC}_{50}$ of $10 \mathrm{nM}$ [8]. Although quercetin in the aglycone form has limited bioavailability in humans $[13,21]$, its presence in the gut may be sufficient to inhibit gut-derived MAO-A in the creation of a designed synergy with a dietary monoamine.

Modulation of the $\mathrm{ABC}$ transporter activity by polyphenols

As discussed above, the ABC transporter family of ATPdriven efflux pumps are responsible for limiting the bioavailability of many xenobiotics and are widespread throughout the body, being especially highly expressed in the epithelia of the gut, placenta and BBB. Figure 2 below shows a pictorial representation of the known members of the $\mathrm{ABC}$ transporters and how they orientate in the gut epithelia.

Cancer cells tend to overexpress the $\mathrm{ABC}$ transporters, conferring greater resistance to chemotherapy agents because these drugs are specifically exported from the cancer cell. This is especially true if a patient is relapsing after a chemotherapy treatment because the remaining cancer cells are being selected for resistance, generally due to the increased expression of the $\mathrm{ABC}$ transporters [49]. Many pharmaceutical $\mathrm{ABC}$ transporter inhibitors are able to decrease drug efflux from cancer cells in vitro, but most tend to have serious and deleterious side effects in vivo, and none is currently approved for inclusion into chemotherapy treatments [19]. The search therefore continues for transporter inhibitors from foods, and especially polyphenol sources, which are expected to have significantly fewer adverse side effects.

Polyphenols have been shown to modulate the activity of some of the $\mathrm{ABC}$ transporters and this effect is believed to be partly responsible for some clinically significant plant-drug interactions. For example, extracts from $\mathrm{St}$ John's Wort (SJW) have been shown to upregulate the expression of intestinal Pgp, that may subsequently reduce the bioavailability of pharmaceuticals that are substrates for this pump. An example of this effect is provided by the antiviral drugs indinavir and saquinavir that are used in the treatment of acquired immune deficiency syndrome (AIDS) [61] and are known Pgp substrates. Treatment with SJW is able to reduce plasma levels of these drugs by up to $57 \%$ in healthy human volunteers, which could potentially lead to subtherapeutic levels and selective pressure for the creation of resistant viral particles in AIDS patients [62]. Recent work by Molnar et al. [49] has shown that a wide range of 
Fig. $2 \mathrm{ABC}$ transporter localisation in gut epithelial cells. P-glycoprotein (Pgp/ MDR1), MRP2 and BCRP are localised in the apical membrane, effluxing compounds back in to the gut lumen; whereas MRP1, 3 and 5 are localised in the basolateral membranes pumping substrates in to the blood stream. MRP4 is present in both the apical and basolateral membranes of gut epithelia [6]

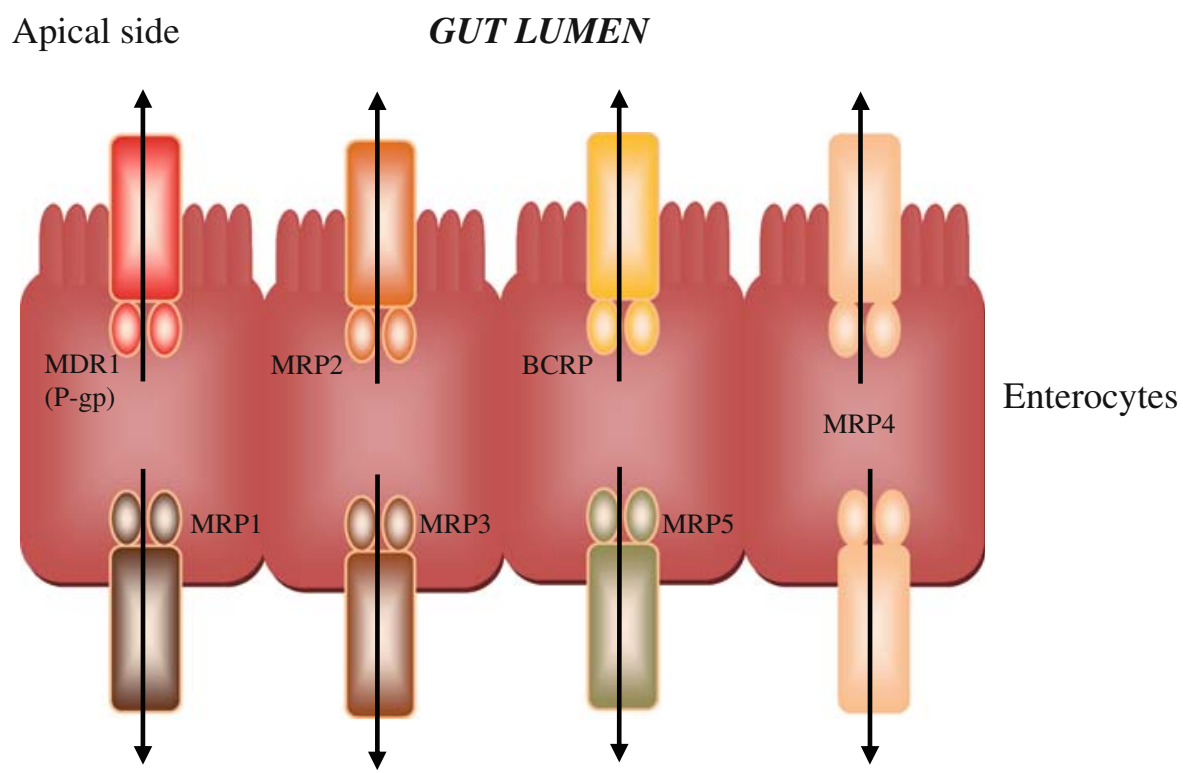

Basolateral side

\section{BLOOD CAPPILARIES}

naturally occurring lipophilic phytochemicals (diterpenes, triterpines and carotenoids) were able to inhibit human Pgp in vitro at the low $\mu \mathrm{g} / \mathrm{ml}$ range, whereas other combinations had positive synergistic activity. Using purified polyphenols on Pgp overexpressing cells in vitro, Patel et al. [59] showed that quercetin, hypericin and kaempferol were able to increase the cellular uptake of ritonavir by five- to eightfold. It is also interesting to note that in vitro assays or short-term exposure to these polyphenols in vivo appears to inhibit the action of efflux pumps and increase substrate bioavailability, whilst chronic exposure in healthy volunteers actually increases the expression of Pgp and, hence, reduces the bioavailability of efflux pump substrate drugs [14, 27].

\section{Polyphenols as $\mathrm{ABC}$ transporter substrates}

Apart from modulating $\mathrm{ABC}$ transporter function, polyphenols can also act as substrates for these efflux pumps, and this property can severely limit their bioavailability. Work by Youdim et al. [89] using an in vivo BBB permeability model showed that the common flavonoid quercetin has very little access through the BBB. Coadministration of quercetin with the Pgp inhibitor PSC833 had no effect on its entry, but co-administration with the BCRP inhibitor GF120918 caused a 20-fold increase in quercetin entry into the brain. These data clearly demonstrate that quercetin is able to enter BBB epithelia, likely by passive diffusion mediated by its hydrophobicity, but is then recognised and specifically exported out of these cells by the BCRP efflux transporter. Likewise, quercetin has very limited bioavailability through gut epithelia and regardless of the amount consumed orally, plasma concentrations of quercetin rarely exceed $1 \mu \mathrm{M}$. Although not specifically proven, this is likely to be at least in part due to BCRP expression in gut epithelia (see [56] for review).

Similarly, the grape polyphenol resveratrol has relatively low oral bioavailability that has recently been shown to be due in part, to the action of the BCRP efflux pump. Transgenic mice deficient in BCRP exhibit greatly increased plasma bioavailability of resveratrol and its metabolites mediated by an enhanced absorption through gut epithelia [80]. Interestingly, not all of the ABC transporters efflux xenobiotics back into the gut lumen after oral consumption (see [6] for review and Fig. 2). For example, the MRP3 transporter pumps xenobiotics into the basolateral side (or blood side) of gut epithelia. Resveratrol is a substrate for this pump, and MRP3-deficient mice have been shown to have significantly decreased plasma levels of resveratrol and its metabolites following the oral consumption [80].

A putative synergy may therefore be designed between a polyphenol $\mathrm{ABC}$ transporter inhibitor and a beneficial polyphenol that has been shown to be a substrate for a particular efflux pump. For example, quercetin bioavailability may be greatly increased when co-consumed with a polyphenol BCRP inhibitor-like apigenin, hesperetin or naringenin [6]. Furthermore, polyphenols which can increase the function or expression of MRP3 activity, thus increasing substrate movement into plasma, may further add to this synergistic interaction. This approach is likely to be more successful when used acutely and short term, as 
chronic exposure to some polyphenol efflux pump inhibitors may actually increase transporter expression over time [14, 27].

Similarly, the beneficial green tea component EGCG and its methyl metabolites have low oral bioavailability, which is thought to be partly due to the actions of the MRP efflux pumps [29, 79]. Co-consumption with a polyphenol MRP2 inhibitor may greatly increase the oral bioavailability of EGCG, and putatively, its beneficial activity on human health. Furthermore, if the efflux pump inhibitors themselves have reasonable oral bioavailability, they may also facilitate the increased penetration through the BBB [90]. For example, quercetin is a weak ligand of the benzodiazepine site of the GABA-A receptor [70] and thus might have anxiolytic activity if it were able to reach its site of action in the brain. The co-consumption of a product rich in quercetin, with a bioavailable BCRP inhibitor may increase quercetin's entry into the brain and hence unleash novel bioactivity from a very commonly consumed polyphenol.

Inhibition of phases 1 and 2 enzymes by polyphenols

The human body is continually exposed to innumerable foreign chemicals, toxins, and phytochemicals through our diet, which, if absorbed, can have a profound influence on our capacity to metabolise xenobiotics, either by inducing the synthesis of more enzyme, or by inhibiting the activity of these enzymes. With regard to polyphenols interacting with these enzymes, probably the best-known example was the discovery in 1991 that the co-administration of certain drugs with grapefruit juice could markedly increase drug bioavailability, often resulting in significant toxicity [2]. Further studies indicated that the main mechanism for this interaction was inhibition of intestinal CYP3A4 by components of grapefruit juice [11]. Of these, the flavonoid naringin and its aglycon metabolite, naringenin have been identified as the most likely inhibitors, but they may not be the sole contributors to this effect. Other flavonoids found in grapefruit juice, such as quercetin, kaemphenol and furanocoumarins have also been shown to cause inhibition of the CYPs in vitro [11, 67, 95]. More recently, evidence is mounting that grapefruit juice can also influence transporter functions, namely by inhibiting the intestinal efflux pump Pgp, and this may play a role in the enhanced oral bioavailability of some drugs [54]. However, as both the CYPs and Pgp share substrate specificity and have overlapping tissue distribution, it is often difficult to differentiate between the two mechanisms [94], and indeed both appear to play complementary roles in limiting the body's exposure to dietary xenobiotics. The best characterised drug-grapefruit juice interactions are those with CYP3A4 substrate drugs with high first-pass metabolism (see [96] for review), including the anxiolytic benzodiazepine family of drugs [22]. Inhibition of CYP3A4 can lead to reduced drug metabolism and greatly increased drug bioavailability, leading to unwanted side effects. For example, the coconsumption of a single serve of grapefruit juice with the benzodiazepine midazolam, increases the area under plasma concentration-time curve (exposure) of midazolam by a factor of 1.65 [22]. Pharmaceuticals that are known to be contraindicated with grapefruit juice contain a warning on their label that grapefruit juice is to be avoided. Functional foods that rely on this effect of grapefruit juice must clearly advertise the fact that their product contains grapefruit juice to prevent unwanted drug interactions.

Although the modulation of CYPs and Pgp by grapefruit juice is the most studied polyphenol-drug interaction, many other polyphenols have been reported to be potent inhibitors of CYPs (mainly from in vitro studies), with possible implications on human therapeutics $[67,95]$. For example, the catechins from green tea, the organosulphur compounds such as diallyl sulphide from garlic, galangin found in honey, sylimarin from milk thistle and other flavonoids, such as diosmetin and luteolin, have all been reported to be inhibitors of various CYPs [7, 65, 67, 95]. There is limited evidence to demonstrate that such in vitro interactions translate to significant in vivo interactions in humans. One study in healthy volunteers who received daidzein, the principal isoflavone in soy bean (200 mg twice daily for 10 days) demonstrated a significant increase in the bioavailability of theophylline, which was thought to be due to the inhibition of CYP1A2, a major enzyme involved in theophylline metabolism [60]. Choi et al. [9] showed that the co-consumption of the immunosuppressant drug cyclosporine with $5 \mathrm{mg} / \mathrm{kg}$ quercetin increased cyclosporine bioavailability by up to $147 \%$ in healthy young volunteers and speculated that this was mediated by an inhibition of the principal metaboliser of cyclosporine, CYP3A4. Similarly, a recent study in rats [51] demonstrated a threefold increase in the oral bioavailability of biochanin A when co-administered with quercetin and EGCG, and speculated that both inhibition of phase 2 metabolism (conjugation by UGT and SULT) and ABC efflux transporters may have contributed to this effect. As noted earlier, polyphenols such as quercetin and EGCG with available functional groups are primarily metabolised by conjugation and it is to be expected that competitive inhibition with other xenobiotic substrates would occur. However, to the best of our knowledge, there are no studies demonstrating inhibition of UGTs or SULTs by polyphenols resulting in clinically relevant pharmacokinetic changes in humans. There are a number of reports indicating that various flavonoids, such as hexamethoxyflavone, tangeretin and silybin are potent inhibitors of UGTs in vitro, with $\mathrm{IC}_{50}$ values $<1 \mu \mathrm{M}[72$, 83, 84]. Similarly, a number of flavonoids (fisetin, galangin, quercetin, myricetin, chrysin, kaempferol, apigenin and 
genistein) have been identified in vitro as potent inhibitors of various SULTs [15, 26, 52], but as with the UGTs, it is not known whether such in vitro interactions translate into significant in vivo effects.

In terms of useful synergies between polyphenols whose bioavailability is limited by phases 1 and 2 enzymes in the creation of a functional food, a study by Fuhr et al. [18] showed that the plasma maximum concentration $\left(C_{\max }\right)$ and half-life of caffeine, which is metabolised by both CYP1A2 and 3A4, could be increased by 23 and $31 \%$, respectively, by the co-consumption with grapefruit juice. However in another similar study, no significant changes in pharmacokinetic parameters were observed and it was suggested that the primary metabolism of caffeine is mediated by CYP1A2, which is not potently affected by grapefruit juice components [40].

\section{Induction of phases 1 and 2 enzymes by polyphenols}

Inhibitory interactions, such as those with grapefruit juice, often result in an exaggerated drug response or even significant toxicity, and thus are usually more easily recognised. However, induction of these same enzymes by xenobiotics, leading to reduced efficacy or therapeutic failure, is less easy to detect. Induction results in a more efficient metabolism and usually reduced bioavailability of compounds that experience significant first-pass metabolism. The best known and most studied induction interaction by polyphenols is that of SJW, a medicinal herb which is widely used to treat depression. A number of case reports and clinical studies have indicated that SJW can precipitate clinically significant and dangerous interactions with certain drugs. For example, co-administration with the immunosuppressant cyclosporin resulted in a significant reduction in cyclosporin bioavailability, causing therapeutic failure and graft rejection [3, 69]. Studies have indicated that the major mechanism underpinning this interaction is induction of CYP3A4, which is the major CYP involved in the phase 1 metabolism of more than $50 \%$ of all the drug/ medicines consumed [95]. In addition, SJW has been demonstrated to increase the expression of Pgp (as well as other transporters), which may contribute to its interactions with structurally diverse drugs [44]. This induction process is thought to be mediated by hyperforin (a phloroglucinol derivative found in SJW) activating the pregnane $\mathrm{X}$ receptor [53]. The latter is a nuclear receptor that regulates the transcription of genes mainly involved in xenobiotic metabolism (e.g., CYPs and UGTs) and disposition (active transporters). However SJW, like most herbal products, contains a large array of biologically active polyphenols (e.g., flavonoids, flavonol glycosides, napthodianthrones and phenylpropanes) with both inhibitory and inductive activity. This may result in confusing and contradictory results depending on the test system used, species, tissue, dose, route and duration of administration, and the source of the SJW. For example, in vitro studies have also indicated that hyperforin is a potent inhibitor of CYP3A4, 2C9 and 2D6 [57]. Other SJW components, such as quercetin and I3, II8-biapigenin have also been reported to inhibit various CYPs [57]. Despite these results, most clinical studies have now confirmed that the administration of SJW for a period of at least 2 weeks significantly reduced plasma concentrations of a large variety of drugs, including oral contraceptives, warfarin, cyclosporin, tacrolimus, verapamil, fexofenadine and SN-38 (the active metabolite of the anticancer drug irinotecan) indicating that the induction of CYPs and perhaps Pgp is the most important reaction from a clinical perspective $[44,53]$.

Apart from SJW, there are a few reported examples of metabolism-related enzyme induction by polyphenols causing a clinically significant effect. Oral administration of a garlic preparation for 3 weeks resulted in a decreased bioavailability of the protease inhibitor saquinavir in healthy subjects [63]. The underlying mechanism was thought to be induction of CYPs, as in vitro studies indicated that the organosulphur compounds from garlic caused induction of CYP1A and 2B, but inhibited CYP2E1 [86] (see [58] for review). Further, tobacco smoke contains polycyclic aromatic hydrocarbons that are known to induce CYP1A2, and the CYP1A2 substrate caffeine is more rapidly cleared in a population of smokers than in nonsmokers [92]. These examples illustrate the problems associated with the extrapolation of possible polyphenoldrug interactions from both in vitro and animal models to humans.

Although induction of phases 1 and 2 metabolic enzymes is usually associated with reduced bioactivity of their substrates, in some instances the metabolite has greater bioactivity than the parent. For example, recent in vitro evidence has shown that phloridzin glucuronide is significantly more potent at protecting human SH-SY5Y neuroblastoma cells from hydrogen peroxide-mediated cell death than the parent molecule phloridzin [74]. Similarly Suri et al. [76] showed that quercetin 3-glucuronide was significantly more potent than quercetin in an in vitro model of inflammation using human neutrophils. Therefore, in this respect the induction of metabolising enzymes leading to greater plasma levels of polyphenolic metabolites may also increase the bioactivity of a functional food ingredient.

Inter-individual variation in polyphenol metabolism and transport

The metabolising enzymes and the efflux transporters in the gut and liver also represent a major factor responsible for the wide variation in a population's response to drugs, 
toxins and polyphenols. For example, 10- to 100 -fold and 30 -fold variations have been reported for CYP3A4 activity in the liver and the small intestine, respectively [39, 81]. Similarly, UGT activity has been shown to vary 6- to 15 fold in human liver microsomes and 10- to 100-fold in intestinal microsomes [78]. Both environmental and genetic factors contribute to this wide variation in the rate and extent of xenobiotic metabolism and disposition between individuals. It is now well recognised that genetic polymorphisms of the xenobiotic-metabolising enzymes and active transport proteins are responsible for a large part of the variable exposure at their site(s) of action, and consequently the response. Most information is available for the CYPs, and genetic polymorphisms have been identified for most CYP isozymes involved in xenobiotic metabolism [31]. CYP2D6 has been most intensively investigated, because its discovery in the mid-seventies, and to date, more than 63 different functional gene variants have been described [31]. Combinations of these variants determine the overall CYP2D6 phenotypes: poor (PM); intermediate (IM); extensive (EM); and ultra-rapid metabolisers (UM). Important interethnic differences in the distribution of the different phenotypes have been observed with PMs (5-12\% of population) mainly found in Europe and UMs (20-28\%) in North Africa and Oceania [31]. Such differences between ethnic populations are particularly relevant to drug therapy, often resulting in adverse reactions and toxicity in PMs, or no response in UMs. Similar phenotypic groups have also been observed with the other CYP systems including, CYP2C9, 2C19 and 2B6 [31]. It is highly likely that these polymorphisms will play a part in the variable efficacy of polyphenols.

Many variants of UGT1A1 and 2B7 have also been identified, but the functional significance of these variants with regard to xenobiotic metabolism has not been well defined [17]. Deficient UGT1A1 is believed to cause-specific genetic conditions, including Gilbert's syndrome, and the more serious and rare Crigler-Najjar syndrome, both characterised by reduced bilirubin glucuronidation, resulting in toxic bilirubin concentrations in blood [17]. Reduced expression of UGT1A1 due to polymorphisms within the gene has also been associated with severe diarrhoea and neutropenia after administration of the anticancer drug irinotecan. The latter is converted to its active metabolite $\mathrm{SN}-38$ which is subsequently conjugated primarily by UGT1A1, for excretion in bile and urine. Increased exposure to $\mathrm{SN}-38$ was responsible for the increased incidence of severe toxicity and pharmacogenetic testing is now used to help avoid this severe toxicity [41]. The variable nature of UGT1A1 due to environmental and genetic factors and its presence in the gut wall and liver suggest that this enzyme could have a significant impact on the bioavailability and exposure of various polyphenols.
Polymorphisms in the $\mathrm{ABC}$ transporters have also been identified, and most studies have focused on Pgp [85]. As indicated previously, the latter is widely distributed in many tissues including the liver, kidney, small intestines, colon, adrenal glands, placenta and the capillary endothelium of the brain and testes. Like the CYPs and UGTs, its expression varies widely between individuals because of environmental and genetic factors. Genetic variations in the ABCB1 gene, which codes for Pgp, have been correlated with drug exposure for a number of commonly used drugs including digoxin, and fenofexadine [28, 36], and it is to be expected that these polymorphisms and their interethnic frequency differences would have a similar impact on the exposure of polyphenols in human populations. This may require, as is the trend in medicinal science, a nutrigenomic approach, where functional foods are tailored to a consumer's-specific genotype.

\section{Modulating-specific transport mechanisms}

Even if a dietary component is bioavailable, it may still not reach its active site in the target organ. For example, dietary tryptophan is an essential amino acid and precursor for the neurotransmitters serotonin and melatonin. Serotonin is not able to cross the BBB and centrally acting serotonin must be synthesised inside the central nervous system [25]. Circulating tryptophan is known to have some access through the BBB via the large neutral amino acid (LNAA) transporter. However, it must compete with other LNAAs for this transporter, and thus the ratio of tryptophan to the other LNAAs in plasma determines its ability to cross the BBB. This ratio can be increased in favour of tryptophan by the co-consumption of a carbohydrate-rich and proteinpoor meal [47]. The mechanism is due to the acute carbohydrate ingestion inducing an insulin spike and the subsequent absorption of LNAAs, except for tryptophan, by muscle cells. Because there is relatively little tryptophan in dietary protein, as compared to the other LNAAs, the lack of dietary protein at the same time will further push the tryptophan/LNAA ratio in favour of tryptophan. Together, this reduces competition for the LNAA transporter, thus allowing a greater influx of tryptophan through the BBB (see [45] for detailed review).

Using the example from above, tryptophan entry through the BBB and into the brain can be increased via the coconsumption of a carbohydrate-rich, protein-poor meal. As mentioned, tryptophan is the precursor to serotonin and increasing tryptophan levels in the brain leads to an increased serotonergic tone and an improvement of symptoms in vulnerable subjects under stress [46]. The production of serotonin from tryptophan first requires a hydroxylase step, forming 5-hydroxytryptophan which is then decarboxylated by the enzyme L-amino acid decarboxylase into serotonin 
[16]. This biosynthetic pathway is analogous to the synthesis of dopamine from its precursor tyrosine. However, neither dopamine nor serotonin has access through the BBB (either in or out of the brain) [25]. To overcome these issues, their respective precursors, which do have some BBB permeability, should be used.

Parkinson's patients who require increased brain levels of dopamine are given the dopamine precursor L-DOPA which unlike dopamine has reasonable entry through the BBB. To prevent L-DOPA from being decarboxylated into dopamine in the periphery, these patients are also given a pharmaceutical decarboxylase inhibitor (usually carbidopa or benserazide), which themselves do not cross the BBB. This decarboxylase inhibitor prevents the peripheral production of dopamine, but does not inhibit the production of dopamine within the brain, and thus allows much greater efficacy of oral L-DOPA therapy [35].

Hypothetically, a complex multi-system synergy could, therefore, be designed exclusively from polyphenols and common foods to increase the production of brain serotonin, which includes:

1. Dietary source of tryptophan (e.g., pumpkin seeds)

2. High carbohydrate load (to increase the tryptophan/ LNAA ratio in favour of tryptophan entry into the brain (e.g., sugar)

3. A peripheral decarboxylase inhibitor which does not cross the BBB to prevent peripheral serotonin production from dietary tryptophan (currently unknown)

4. A centrally acting MAO inhibitor which may modulate serotonin deamination within the brain (e.g., Ginkgo biloba extract [68]).

\section{Conclusion}

In this review and hypothesis paper, the primary mechanisms that limit the bioavailability of both food-derived polyphenols and pharmaceutical drugs have been discussed. Within the functional food research arena, many bioactive polyphenols and crude plant extracts have been identified, but few have shown true efficacy in strictly designed clinical trials, primarily because of poor bioavailability of the bioactive ingredients. It has also been proposed that clinical trials, as used in pharmaceutical research, may not be appropriate for functional foods, because as the name suggests, these are foods and not drugs. The activity of a functional food is generally not expected to be acute and potent, but rather cumulative and subtle. Further, during drug trials, participants are known to be free of the drug under investigation and will only receive the experimental drug during the trial. This is clearly not the case in functional food trials as participants generally have varied diets before, during and after the trial which can significantly affect outcome, including by some of the mechanisms discussed here.

Research on polyphenol-drug interactions has illustrated the ability of some plant compounds to significantly affect the bioavailability and activity of pharmaceutical agents, usually resulting in adverse effects. However, it seems that these interactions can be utilised to designspecific synergies between polyphenols to potently increase their bioactivity, and hence, their beneficial health-promoting effects.

However, great care must be taken to avoid potentially deleterious drug-food and food-food interactions, such as in the MAO inhibitor-dietary tyramine interaction mentioned earlier. Where unavoidable, these interactions must be minimised and clearly advertised to consumers. In reality, polyphenols are xenobiotics and are handled by the body in a similar manner as any synthetic or non-physiological agent or drug. Their apparent lack of toxicity may just reflect their lower exposure level, rather than any intrinsic safety. Given these limitations, we propose that the "designed synergy" strategies discussed here offer an exciting new tool for the creation of future functional foods.

Acknowledgments The authors would like to thank Dr D. Stevenson, Mr E. Walker, Dr M.A. Skinner and Dr J. Ingram for reading and commenting on the manuscript. This work was funded by a capability fund (CF33) from The New Zealand Institute for Plant and Food Research Limited.

\section{References}

1. Andreasen MF, Kroon PA, Williamson G, Garcia-Conesa MT (2001) Esterase activity able to hydrolyze dietary antioxidant hydroxycinnamates is distributed along the intestine of mammals. J Agric Food Chem 49:5679-5684

2. Bailey DG, Spence JD, Munoz C, Arnold JM (1991) Interaction of citrus juices with felodipine and nifedipine. Lancet 337:268269

3. Barone GW, Gurley BJ, Ketel BL, Lightfoot ML, Abul-Ezz SR (2000) Drug interaction between St. John's wort and cyclosporine. Ann Pharmacother 34:1013-1016

4. Bormann J, Demisch L, Gurtelmeyer R, Koch R, Schatton W (1991) Cerebral-activating extract. US Patent 755814

5. Bouayed J, Rammal H, Dicko A, Younos C, Soulimani R (2007) Chlorogenic acid, a polyphenol from Prunus domestica (Mirabelle), with coupled anxiolytic and antioxidant effects. J Neurol Sci 262:77-84

6. Brand W, Schutte ME, Williamson G, van Zanden JJ, Cnubben $\mathrm{NH}$, Groten JP, van Bladeren PJ, Rietjens IM (2006) Flavonoidmediated inhibition of intestinal $\mathrm{ABC}$ transporters may affect the oral bioavailability of drugs, food-borne toxic compounds and bioactive ingredients. Biomed Pharmacother 60:508-519

7. Butterweck V, Derendorf H (2008) Potential of pharmacokinetic profiling for detecting herbal interactions with drugs. Clin Pharmacokinet 47:383-397

8. Chimenti F, Cottiglia F, Bonsignore L, Casu L, Casu M, Floris C, Secci D, Bolasco A, Chimenti P, Granese A, Befani O, Turini P, 
Alcaro S, Ortuso F, Trombetta G, Loizzo A, Guarino I (2006) Quercetin as the active principle of Hypericum hircinum exerts a selective inhibitory activity against MAO-A: extraction, biological analysis, and computational study. J Nat Prod 69:945-949

9. Choi JS, Choi BC, Choi KE (2004) Effect of quercetin on the pharmacokinetics of oral cyclosporine. Am J Health Syst Pharm 61:2406-2409

10. Crespy V, Morand C, Besson C, Manach C, Demigne C, Remesy C (2002) Quercetin, but not its glycosides, is absorbed from the rat stomach. J Agric Food Chem 50:618-621

11. Dahan A, Altman H (2004) Food-drug interaction: grapefruit juice augments drug bioavailability-mechanism, extent and relevance. Eur J Clin Nutr 58:1-9

12. Day AJ, Bao YP, Morgan MRA, Williamson G (2000) Conjugation position of quercetin glucuronides and effect on biological activity. Free Radic Biol Med 29:1234-1243

13. Day AJ, Mellon F, Barron D, Sarrazin G, Morgan MR, Williamson G (2001) Human metabolism of dietary flavonoids: identification of plasma metabolites of quercetin. Free Radic Res 35:941-952

14. Durr D, Stieger B, Kullak-Ublick GA, Rentsch KM, Steinert HC, Meier PJ, Fattinger K (2000) St John's Wort induces intestinal Pglycoprotein/MDR1 and intestinal and hepatic CYP3A4. Clin Pharmacol Ther 68:598-604

15. Eaton EA, Walle UK, Lewis AJ, Hudson T, Wilson AA, Walle T (1996) Flavonoids, potent inhibitors of the human P-form phenolsulfotransferase: potential role in drug metabolism and chemoprevention. Drug Metab Dispos 24:232-237

16. Fernstrom JD (1990) Aromatic amino acids and monoamine synthesis in the central nervous system: influence of the diet. J Nutr Biochem 1:508-517

17. Fisher MB, Paine MF, Strelevitz TJ, Wrighton SA (2001) The role of hepatic and extrahepatic UDP-glucuronosyltransferases in human drug metabolism. Drug Metab Rev 33:273-297

18. Fuhr U, Klittich K, Staib AH (1993) Inhibitory effect of grapefruit juice and its bitter principal, naringenin, on CYP1A2 dependent metabolism of caffeine in man. Br J Clin Pharmacol 35:431-436

19. Gandhi L, Harding MW, Neubauer M, Langer CJ, Moore M, Ross HJ, Johnson BE, Lynch TJ (2007) A phase II study of the safety and efficacy of the multidrug resistance inhibitor VX-710 combined with doxorubicin and vincristine in patients with recurrent small cell lung cancer. Cancer 109:924-932

20. Gardner DM, Shulman KI, Walker SE, Tailor SA (1996) The making of a user friendly MAOI diet. J Clin Psychiatry 57:99_ 104

21. Graefe EU, Wittig J, Mueller S, Riethling AK, Uehleke B, Drewelow B, Pforte H, Jacobasch G, Derendorf H, Veit M (2001) Pharmacokinetics and bioavailability of quercetin glycosides in humans. J Clin Pharmacol 41:492-499

22. Greenblatt DJ, von Moltke LL, Harmatz JS, Chen G, Weemhoff JL, Jen C, Kelley CJ, LeDuc BW, Zinny MA (2003) Time course of recovery of cytochrome p450 3A function after single doses of grapefruit juice. Clin Pharmacol Ther 74:121-129

23. Halberstadt AL, Buell MR, Masten VL, Risbrough VB, Geyer MA (2008) Modification of the effects of 5-methoxy- $N, \mathrm{~N}$ dimethyltryptamine on exploratory behavior in rats by monoamine oxidase inhibitors. Psychopharmacology (Berl) 201:55-66

24. Han XH, Hong SS, Hwang JS, Lee MK, Hwang BY, Ro JS (2007) Monoamine oxidase inhibitory components from Cayratia japonica. Arch Pharm Res 30:13-17

25. Hardebo JE, Owman C (1980) Barrier mechanisms for neurotransmitter monoamines and their precursors at the blood-brain interface. Ann Neurol 8:1-31

26. Harris RM, Wood DM, Bottomley L, Blagg S, Owen K, Hughes PJ, Waring RH, Kirk CJ (2004) Phytoestrogens are potent inhibitors of estrogen sulfation: implications for breast cancer risk and treatment. J Clin Endocrinol Metab 89:1779-1787

27. Hennessy M, Kelleher D, Spiers JP, Barry M, Kavanagh P, Back D, Mulcahy F, Feely J (2002) St Johns wort increases expression of P-glycoprotein: implications for drug interactions. Br J Clin Pharmacol 53:75-82

28. Hoffmeyer S, Burk O, von Richter O, Arnold HP, Brockmoller J, Johne A, Cascorbi I, Gerloff T, Roots I, Eichelbaum M, Brinkmann U (2000) Functional polymorphisms of the human multidrug-resistance gene: multiple sequence variations and correlation of one allele with P-glycoprotein expression and activity in vivo. Proc Natl Acad Sci USA 97:3473-3478

29. Hong J, Lambert JD, Lee SH, Sinko PJ, Yang CS (2003) Involvement of multidrug resistance-associated proteins in regulating cellular levels of (-)-epigallocatechin-3-gallate and its methyl metabolites. Biochem Biophys Res Commun 310:222227

30. Hu M, Chen J, Lin H (2003) Metabolism of flavonoids via enteric recycling: mechanistic studies of disposition of apigenin in the Caco-2 cell culture model. J Pharmacol Exp Ther 307:314-321

31. Ingelman-Sundberg M, Sim SC, Gomez A, Rodriguez-Antona C (2007) Influence of cytochrome P450 polymorphisms on drug therapies: pharmacogenetic, pharmacoepigenetic and clinical aspects. Pharmacol Ther 116:496-526

32. Janisch KM, Williamson G, Needs P, Plumb GW (2004) Properties of quercetin conjugates: modulation of LDL oxidation and binding to human serum albumin. Free Radic Res 38:877-884

33. Jovanovich SV, Steenken S, Simic MG, Hara Y (1998) Flavonoids in health and disease. In: Rice-Evans C, Packer L (eds) Flavonoids in health and disease. Marcel Dekker, NY, pp 137-161

34. Kennedy DO, Pace S, Haskell C, Okello EJ, Milne A, Scholey $\mathrm{AB}$ (2006) Effects of cholinesterase inhibiting sage (Salvia officinalis) on mood, anxiety and performance on a psychological stressor battery. Neuropsychopharmacology 31:845-852

35. Khor SP, Hsu A (2007) The pharmacokinetics and pharmacodynamics of levodopa in the treatment of Parkinson's disease. Curr Clin Pharmacol 2:234-243

36. Kim RB, Leake BF, Choo EF, Dresser GK, Kubba SV, Schwarz UI, Taylor A, Xie HG, McKinsey J, Zhou S, Lan LB, Schuetz JD, Schuetz EG, Wilkinson GR (2001) Identification of functionally variant MDR1 alleles among European Americans and African Americans. Clin Pharmacol Ther 70:189-199

37. Lambert JD, Sang SM, Yang CS (2007) Biotransformation of green tea polyphenols and the biological activities of those metabolites. Mol Pharmaceutics 4:819-825

38. Lewis DF, Ito $\mathrm{Y}$ (2008) Human cytochromes P450 in the metabolism of drugs: new molecular models of enzyme-substrate interactions. Expert Opin Drug Metab Toxicol 4:1181-1186

39. Lown KS, Kolars JC, Thummel KE, Barnett JL, Kunze KL, Wrighton SA, Watkins PB (1994) Interpatient heterogeneity in expression of CYP3A4 and CYP3A5 in small bowel: lack of prediction by the erythromycin breath test. Drug Metab Dispos 22:947-955

40. Maish WA, Hampton EM, Whitsett TL, Shepard JD, Lovallo WR (1996) Influence of grapefruit juice on caffeine pharmacokinetics and pharmacodynamics. Pharmacotherapy 16:1046-1052

41. Maitland ML, DiRienzo A, Ratain MJ (2006) Interpreting disparate responses to cancer therapy: the role of human population genetics. J Clin Oncol 24:2151-2157

42. Manach C, Scalbert A, Morand C, Remesy C, Jimenez L (2004) Polyphenols: food sources and bioavailability. Am J Clin Nutr 79:727-747

43. Manach C, Williamson G, Morand C, Scalbert A, Remesy C (2005) Bioavailability and bioefficacy of polyphenols in humans. I. Review of 97 bioavailability studies. Am J Clin Nutr 81:230S$242 \mathrm{~S}$ 
44. Markowitz JS, Donovan JL, DeVane CL, Taylor RM, Ruan Y, Wang JS, Chavin KD (2003) Effect of St John's wort on drug metabolism by induction of cytochrome P450 3A4 enzyme. JAMA 290:1500-1504

45. Markus CR (2008) Dietary amino acids and brain serotonin function: implications for stress-related affective changes. Neuromolecular Med 10(4):247-258

46. Markus CR, Olivier B, Panhuysen GE, Van Der Gugten J, Alles MS, Tuiten A, Westenberg HG, Fekkes D, Koppeschaar HF, de Haan EE (2000) The bovine protein alpha-lactalbumin increases the plasma ratio of tryptophan to the other large neutral amino acids, and in vulnerable subjects raises brain serotonin activity, reduces cortisol concentration, and improves mood under stress. Am J Clin Nutr 71:1536-1544

47. Markus R, Panhuysen G, Tuiten A, Koppeschaar H (2000) Effects of food on cortisol and mood in vulnerable subjects under controllable and uncontrollable stress. Physiol Behav 70:333-342

48. McKenna DJ, Towers GH, Abbott F (1984) Monoamine oxidase inhibitors in South American hallucinogenic plants: tryptamine and beta-carboline constituents of ayahuasca. J Ethnopharmacol 10:195-223

49. Molnar J, Gyemant N, Tanaka M, Hohmann J, Bergmann-Leitner E, Molnar P, Deli J, Didiziapetris R, Ferreira MJ (2006) Inhibition of multidrug resistance of cancer cells by natural diterpenes, triterpenes and carotenoids. Curr Pharm Des 12:287-311

50. Monteiro M, Farah A, Perrone D, Trugo LC, Donangelo C (2007) Chlorogenic acid compounds from coffee are differentially absorbed and metabolized in humans. J Nutr 137:2196-2201

51. Moon YJ, Morris ME (2007) Pharmacokinetics and bioavailability of the bioflavonoid biochanin A: effects of quercetin and EGCG on biochanin A disposition in rats. Mol Pharm 4:865-872

52. Moon YJ, Wang X, Morris ME (2006) Dietary flavonoids: effects on xenobiotic and carcinogen metabolism. Toxicol In Vitro 20:187-210

53. Moore LB, Goodwin B, Jones SA, Wisely GB, Serabjit-Singh CJ, Willson TM, Collins JL, Kliewer SA (2000) St. John's wort induces hepatic drug metabolism through activation of the pregnane X receptor. Proc Natl Acad Sci USA 97:7500-7502

54. Morris ME, Zhang S (2006) Flavonoid-drug interactions: effects of flavonoids on ABC transporters. Life Sci 78:2116-2130

55. Mukherjee PK, Kumar V, Mal M, Houghton PJ (2007) Acetylcholinesterase inhibitors from plants. Phytomedicine 14:289-300

56. Murakami T, Takano M (2008) Intestinal efflux transporters and drug absorption. Expert Opin Drug Metab Toxicol 4:923-939

57. Obach RS (2000) Inhibition of human cytochrome P450 enzymes by constituents of St. John's Wort, an herbal preparation used in the treatment of depression. J Pharmacol Exp Ther 294:88-95

58. Pal D, Mitra AK (2006) MDR- and CYP3A4-mediated drug-drug interactions. J Neuroimmune Pharmacol 1:323-339

59. Patel J, Buddha B, Dey S, Pal D, Mitra AK (2004) In vitro interaction of the HIV protease inhibitor ritonavir with herbal constituents: changes in P-gp and CYP3A4 activity. Am J Ther 11:262-277

60. Peng WX, Li HD, Zhou HH (2003) Effect of daidzein on CYP1A2 activity and pharmacokinetics of theophylline in healthy volunteers. Eur J Clin Pharmacol 59:237-241

61. Perloff MD, von Moltke LL, Stormer E, Shader RI, Greenblatt DJ (2001) Saint John's wort: an in vitro analysis of P-glycoprotein induction due to extended exposure. Br J Pharmacol 134:16011608

62. Piscitelli SC, Burstein AH, Chaitt D, Alfaro RM, Falloon J (2000) Indinavir concentrations and St John's wort. Lancet 355:547-548

63. Piscitelli SC, Burstein AH, Welden N, Gallicano KD, Falloon J (2002) The effect of garlic supplements on the pharmacokinetics of saquinavir. Clin Infect Dis 34:234-238
64. Pittler MH, Ernst E (2003) Kava extract for treating anxiety. Cochrane Database Syst Rev 1:CD003383

65. Quintieri L, Palatini P, Nassi A, Ruzza P, Floreani M (2008) Flavonoids diosmetin and luteolin inhibit midazolam metabolism by human liver microsomes and recombinant CYP 3A4 and CYP3A5 enzymes. Biochem Pharmacol 75:1426-1437

66. Radtke J, Linseisen J, Wolfram G (1998) Phenolic acid intake of adults in a Bavarian subgroup of the national food consumption survey. Z Ernahrungswiss 37:190-197

67. Rodeiro I, Donato MT, Lahoz A, Garrido G, Delgado R, GomezLechon MJ (2008) Interactions of polyphenols with the P450 system: possible implications on human therapeutics. Mini Rev Med Chem 8:97-106

68. Rojas P, Rojas C, Ebadi M, Montes S, Monroy-Noyola A, Serrano-Garcia N (2004) EGb761 pretreatment reduces monoamine oxidase activity in mouse corpus striatum during 1-methyl-4phenylpyridinium neurotoxicity. Neurochem Res 29:1417-1423

69. Ruschitzka F, Meier PJ, Turina M, Luscher TF, Noll G (2000) Acute heart transplant rejection due to Saint John's wort. Lancet 355:548-549

70. Salgueiro JB, Ardenghi P, Dias M, Ferreira MB, Izquierdo I, Medina JH (1997) Anxiolytic natural and synthetic flavonoid ligands of the central benzodiazepine receptor have no effect on memory tasks in rats. Pharmacol Biochem Behav 58:887-891

71. Sousa T, Paterson R, Moore V, Carlsson A, Abrahamsson B, Basit AW (2008) The gastrointestinal microbiota as a site for the biotransformation of drugs. Int J Pharm 363:1-25

72. Sridar C, Goosen TC, Kent UM, Williams JA, Hollenberg PF (2004) Silybin inactivates cytochromes P450 3A4 and 2C9 and inhibits major hepatic glucuronosyltransferases. Drug Metab Dispos 32:587-594

73. Stein GE, Gurwith MJ (1984) Amoxicillin potassium clavulanate, a beta-lactamase-resistant antibiotic combination. Clin Pharm 3:591-599

74. Stevenson DE, Cooney JM, Jensen DJ, Wibisono R, Adaim A, Skinner MA, Zhang J (2008) Comparison of enzymically glucuronidated flavonoids with flavonoid aglycones in an in vitro cellular model of oxidative stress protection. In Vitro Cell Dev Biol Anim 44:73-80

75. Stevenson DE, Hurst RD (2007) Polyphenolic phytochemicalsjust antioxidants or much more? Cell Mol Life Sci 64:2900-2916

76. Suri S, Taylor MA, Verity A, Tribolo S, Needs PW, Kroon PA, Hughes DA, Wilson VG (2008) A comparative study of the effects of quercetin and its glucuronide and sulfate metabolites on human neutrophil function in vitro. Biochem Pharmacol 76:645-653

77. Tildesley NT, Kennedy DO, Perry EK, Ballard CG, Savelev S, Wesnes KA, Scholey AB (2003) Salvia lavandulaefolia (Spanish sage) enhances memory in healthy young volunteers. Pharmacol Biochem Behav 75:669-674

78. Urquhart BL, Tirona RG, Kim RB (2007) Nuclear receptors and the regulation of drug-metabolizing enzymes and drug transporters: implications for interindividual variability in response to drugs. J Clin Pharmacol 47:566-578

79. Vaidyanathan JB, Walle T (2003) Cellular uptake and efflux of the tea flavonoid (-)epicatechin-3-gallate in the human intestinal cell line Caco-2. J Pharmacol Exp Ther 307:745-752

80. van de Wetering K, Burkon A, Feddema W, Bot A, de Jonge H, Somoza V, Borst P (2008) Intestinal BCRP/Bcrp1 and MRP3/ Mrp3 are involved in the pharmacokinetics of resveratrol. Mol Pharmacol 75(4):876-885

81. Wacher VJ, Silverman JA, Zhang Y, Benet LZ (1998) Role of Pglycoprotein and cytochrome P450 3A in limiting oral absorption of peptides and peptidomimetics. J Pharm Sci 87:1322-1330

82. Walle T, Browning AM, Steed LL, Reed SG, Walle UK (2005) Flavonoid glucosides are hydrolyzed and thus activated in the oral cavity in humans. J Nutr 135:48-52 
83. Williams JA, Hyland R, Jones BC, Smith DA, Hurst S, Goosen TC, Peterkin V, Koup JR, Ball SE (2004) Drug-drug interactions for UDP-glucuronosyltransferase substrates: a pharmacokinetic explanation for typically observed low exposure (AUCi/AUC) ratios. Drug Metab Dispos 32:1201-1208

84. Williams JA, Ring BJ, Cantrell VE, Campanale K, Jones DR, Hall SD, Wrighton SA (2002) Differential modulation of UDPglucuronosyltransferase 1A1 (UGT1A1)-catalyzed estradiol-3glucuronidation by the addition of UGT1A1 substrates and other compounds to human liver microsomes. Drug Metab Dispos 30:1266-1273

85. Woodahl EL, Ho RJ (2004) The role of MDR1 genetic polymorphisms in interindividual variability in P-glycoprotein expression and function. Curr Drug Metab 5:11-19

86. Wu CC, Sheen LY, Chen HW, Kuo WW, Tsai SJ, Lii CK (2002) Differential effects of garlic oil and its three major organosulfur components on the hepatic detoxification system in rats. J Agric Food Chem 50:378-383

87. Xing J, Chen X, Zhong D (2005) Absorption and enterohepatic circulation of baicalin in rats. Life Sci 78:140-146

88. Yang CS, Lambert JD, Ju J, Lu G, Sang S (2007) Tea and cancer prevention: molecular mechanisms and human relevance. Toxicol Appl Pharmacol 224:265-273
89. Youdim KA, Dobbie MS, Kuhnle G, Proteggente AR, Abbott NJ, Rice-Evans C (2003) Interaction between flavonoids and the blood-brain barrier: in vitro studies. J Neurochem 85:180-192

90. Youdim KA, Qaiser MZ, Begley DJ, Rice-Evans CA, Abbott NJ (2004) Flavonoid permeability across an in situ model of the blood-brain barrier. Free Radic Biol Med 36:592-604

91. Youdim MB, Edmondson D, Tipton KF (2006) The therapeutic potential of monoamine oxidase inhibitors. Nat Rev Neurosci 7:295-309

92. Zevin S, Benowitz NL (1999) Drug interactions with tobacco smoking: an update. Clin Pharmacokinet 36:425-438

93. Zhang L, Zuo Z, Lin G (2007) Intestinal and hepatic glucuronidation of flavonoids. Mol Pharm 4:833-845

94. Zhang YC, Benet LZ (2001) The gut as a barrier to drug absorption-combined role of cytochrome P450 3A and P-glycoprotein. Clin Pharmacokinet 40:159-168

95. Zhou S, Gao Y, Jiang W, Huang M, Xu A, Paxton JW (2003) Interactions of herbs with cytochrome P450. Drug Metab Rev 35:35-98

96. Zhou S, Lim LY, Chowbay B (2004) Herbal modulation of Pglycoprotein. Drug Metab Rev 36:57-104 